\title{
Demonstration of three dopamine molecules bound to $\alpha$-synuclein: Implication of oligomerization at the initial stage
}

\author{
Sakurako Shimotakahara ${ }^{1^{*}}$, Yuuki Shiroyama ${ }^{2}$, Takashi Fujimoto ${ }^{3}$, Mai Akai ${ }^{1}$, Takaya Onoue ${ }^{1}$, \\ Hiroko Seki ${ }^{4}$, Sayaka Kado ${ }^{4}$, Tomoya Machinami ${ }^{3}$, Yoichi Shibusawa ${ }^{1}$, Kenji Uéda ${ }^{5}$, \\ Mitsuru Tashiro ${ }^{3}$ \\ ${ }^{1}$ School of Pharmacy, Tokyo University of Pharmacy and Life Science, Tokyo, Japan; \\ *Corresponding Author: tashiro@toyaku.ac.jp \\ ${ }^{2}$ School of Life Science, Tokyo University of Pharmacy and Life Science, Tokyo, Japan \\ ${ }^{3}$ Department of Chemistry, School of Sciences and Engineering, Meisei University, Tokyo, Japan \\ ${ }^{4}$ Chemical Analysis Center, Chiba University, Chiba, Japan \\ ${ }^{5}$ Department of Dementia and Higher Brain Function, Tokyo Metropolitan Institute of Medical Science, Tokyo, Japan
}

Received 6 January 2012; revised 25 January 2012; accepted 17 February 2012

\begin{abstract}
$\alpha$-Synuclein is the major component of the filamentous Lewy bodies and Lewy neurites that define neuropathological features and dementia with Lewy bodies. To investigate the role of dopamine (DA) in $\alpha$-synuclein fibrillation, the structural propensities to form oligomers at the initial stage fibrillation were studied using size exclusion chromatography and various biophysical techniques. Interactions with DA were observed for wild-type $\alpha$-synuclein and its mutants, A30P, E46K and A53T, using electrospray ionization mass spectrometry (ESI-MS). The results of ESIMS indicate that an intact $\alpha$-synuclein, which was not oxidized, had an ability to bind with three molecules of DA at the initial stage. Furthermore, upon binding to DA, $\alpha$-synuclein oligomerizes to higher molecular weight species. These oligomers are structurally different from amyloid fibrils, as confirmed by thioflavin T and CD analysis.
\end{abstract}

Keywords: $\alpha$-Synuclein; Dopamine; Fibrillation; Oligomerization; Mass Spectrometry

\section{INTRODUCTION}

$\alpha$-Synuclein is a 140 -amino acid protein characterized by an acidic C-terminal region and seven imperfect repeats (consensus KTKEGV) distributed throughout most of the N-terminal half of the polypeptide [1]. $\alpha$-Synuclein is a highly soluble, heat-stable and natively unfolded protein $[2,3]$ predominantly expressed in the neurons of the central nervous system. It is localized at presynaptic terminals in close proximity to synaptic vesicles $[1,4-6]$, as well as in the neuronal nuclei [7]. Three different missense mutations in the $\alpha$-synuclein gene, A53T [8], A30P [9], and E46K [10], were identified in the confirmed familial cases of autosomal-dominantly inherited, early-onset Parkinson's disease (PD) and dementia with Lewy bodies. It has been observed that $\alpha$-synuclein accumulated in Lewy bodies and Lewy-related neurites mainly forms fibrils in PD [11]. The previous studies [12-17] indicated that the hydrophobic repeat region between residues 65 and 95 within the non- $\mathrm{A} \beta$ component of Alzheimer's disease amyloid (NAC) region and the negative charges at the C-terminal region played a key role in forming fibril structures. Molecular species that were relatively compact but still unfolded were also found to be involved in the fibril formation.

Recently, interactions between $\alpha$-synuclein and dopamine (DA) have been vigorously studied [18-22]. These studies suggest that distinctly reactive intermediates of $\alpha$-synuclein interacting with DA induce its oligomerization [20]. Although it has been proposed that the formation of DA-interacting $\alpha$-synuclein provides an explanation for the dopaminergic pathway of $\alpha$-synuclein-associated neurotoxicity in PD [18], the details of interactions between $\alpha$-synuclein and DA are yet to be elucidated. Our group has been engaging in the study of fibrillation process of $\alpha$-synuclein at the initial stage. The singular value decomposition analysis using the time-dependent CD spectra revealed that five or nine intermediates were formed at the early stage of fibrillation [23]. In our timedependent small angle X-ray scattering (SAXS) measurements, formation of oligomers comprising heptamer was 
suggested [24]. In order to investigate the detailed oligomer association mechanism of $\alpha$-synuclein at the initial stage, interactions between $\alpha$-synuclein and DA have been studied using various biochemical and biophysical techniques. The measurements using an electrospray ionization mass spectrometry (ESI-MS), size exclusion chromatography, fluorescence and $\mathrm{CD}$ have been carried out for the wild-type $\alpha$-synuclein and three mutants, A30P, E46K and A53T, to evaluate the effect of these mutations in oligomerization. Although mechanisms that govern the formation of $\alpha$-synuclein aggregates and oligomers are not well understood, they are considered to be a central event in the pathogenesis of PD. Moreover, we consider DA to be a one of the critical factors for aggregation or oligomerization event. Hence, elucidation of structural features of $\alpha$-synuclein oligomerization with DA is expected to provide significantly important clues for the molecular mechanism of PD.

\section{MATERIALS AND METHODS}

\subsection{Preparation of Oligomers and ThT Measurements}

The wild-type $\alpha$-synuclein and three mutants, A30P, E46K and A53T, were cloned, expressed and purified as described previously [23]. $\alpha$-Synuclein (ca. $2.0 \mathrm{mg} / \mathrm{ml}$ ) was assembled into oligomers and/or fibrils in the absence and presence of DA $(0.1-10 \mathrm{mM})$ by incubating in 10 $\mathrm{mM}$ ammonium acetate $(\mathrm{pH} 7.5)$ at $30^{\circ} \mathrm{C}$ with continuous stirring at $450 \mathrm{rpm}$. Thioflavin-T (ThT) fluorescence intensities were measured every $15 \mathrm{~min}$ at $482 \mathrm{~nm}$ with excitation at $450 \mathrm{~nm}$ and 100 scans on a microplate reader SH-9000Lab (Corona Electric).

\subsection{Spectroscopy}

CD spectra were recorded using a 1-mm cuvette, scanning from 190 to $250 \mathrm{~nm}$ with a step size of $0.1 \mathrm{~nm}$ and scanning speed of $50 \mathrm{~nm} / \mathrm{min}$ on a Jasco J-720 spectrophotometer. For all spectra, an average of eight scans was obtained every 24 hours. CD samples were prepared by diluting $10 \mu \mathrm{L}$ of the above protein sample $(2.0 \mathrm{mg} / \mathrm{ml})$ at various incubation times into $400 \mu \mathrm{L} \mathrm{CD}$ buffer (10 $\mathrm{mM}$ sodium phosphate, $\mathrm{pH} 7.5$ ), resulting in a final protein concentration of $0.05 \mathrm{mg} / \mathrm{ml}$.

\subsection{MS Spectrometry}

Measurements of FT-MS were carried out for A30P using a LTQ-Orbitrap XL FT-mass spectrometer (Thermo Scientific). The experimental conditions were as follows: desolvation temperature, $200^{\circ} \mathrm{C}$; sample flow rate, 20 $\mu \mathrm{L} / \mathrm{min} ; R=15000$. ESI-MS measurements were carried out for the wild-type, E46K and A53T $\alpha$-synucleins using a time-of-flight mass spectrometer (JEOL JMS-T100).
The experimental conditions were as follows: acceleration voltage, $2.0 \mathrm{kV}$; needle voltage, $2600 \mathrm{~V}$; orifice voltage, $65 \mathrm{~V}$; desolvation temperature, $100^{\circ} \mathrm{C}$; sample flow rate, $5.0 \mu \mathrm{L} / \mathrm{min}$. All spectra were obtained via infusion of the sample solutions in the positive ionization mode.

\subsection{Size Exclusion Chromatography}

The incubated sample solutions were filtrated through a membrane filter Dismic-03 (pore size, $0.45 \mu \mathrm{m}$; Toyo Roshi), and then applied onto a TSKgel G3000SW $\mathrm{XL}_{\mathrm{XL}}$ gel filtration column $(7.8 \mathrm{~mm}$ I.D. $\times 300 \mathrm{~mm}$; Tosoh Bioscience) with $50 \mathrm{mM}$ sodium phosphate buffer, $\mathrm{pH} 7.5$, containing $100 \mathrm{mM} \mathrm{NaCl}$. The flow rate was $0.4 \mathrm{~mL} / \mathrm{min}$.

\section{RESULTS AND DISCUSSION}

\subsection{Effects of Dopamine in Amyloid Fibril Formation}

In order to observe the effects of DA in amyloid fibril formation of $\alpha$-synucleins, an accumulation of amyloid was monitored by fluorescence spectroscopy using a ThT binding assay. This method is commonly used to observe an amyloid fibril formation of proteins. In general, an increase in ThT fluorescence intensity is considered to be a good indication of filament accumulations [25]. The time-dependent changes of ThT fluorescence intensity in the presence and absence of DA are shown in Figure 1. The striking decreases of ThT fluorescence intensities in the presence of $1 \mathrm{mM}$ DA were observed for the wildtype, A30P and A53T $\alpha$-synucleins. The ThT fluorescence intensities of E46K in the presence of $1 \mathrm{mM}$ DA were decreased more than $50 \%$ of those in the absence of DA. These results indicate that DA significantly inhibited the amyloid fibril formation of the wild-type $\alpha$-synuclein and its three mutants. Almost no fibril was observed in the wild-type and A30P $\alpha$-synucleins up to the incubation time of 100 hours (Figure 1(B)).

\section{2. $\beta$-Sheet Contents; Characterization by CD}

Figure 2 shows the time courses of changes in molar ellipticities at $218 \mathrm{~nm}$ for the wild-type $\alpha$-synuclein and its mutants. The amounts of $\beta$-sheet structure can be estimated by the negative ellipticity at $218 \mathrm{~nm}$. The overall time dependent changes of ellipticities in the presence and absence of DA were similar for the wild-type, E46K and A53T $\alpha$-synucleins, and the values of ellipticity was slightly higher for A30P in the presence of $1 \mathrm{mM}$ DA. The results of $\mathrm{CD}$ spectra indicate that $\beta$-sheet contents were almost unaffected by the presence of DA for the wildtype, E46K and A53T $\alpha$-synucleins, whereas slight decrease was observed for A30P in the presence of $1 \mathrm{mM}$ DA. Because fibril formation of all mutants were suppressed 


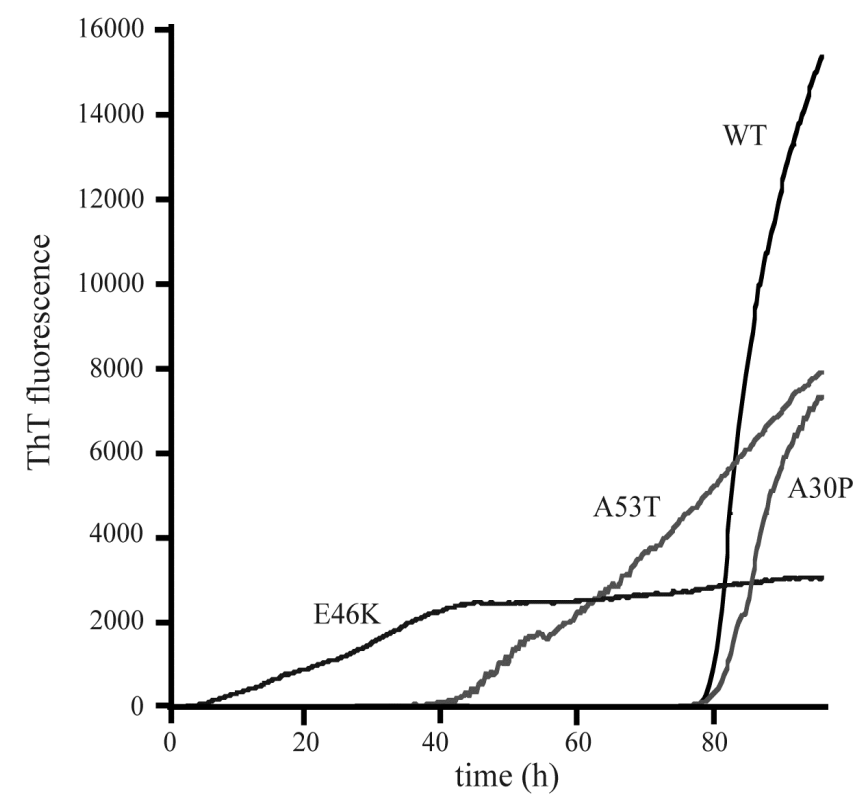

(a)

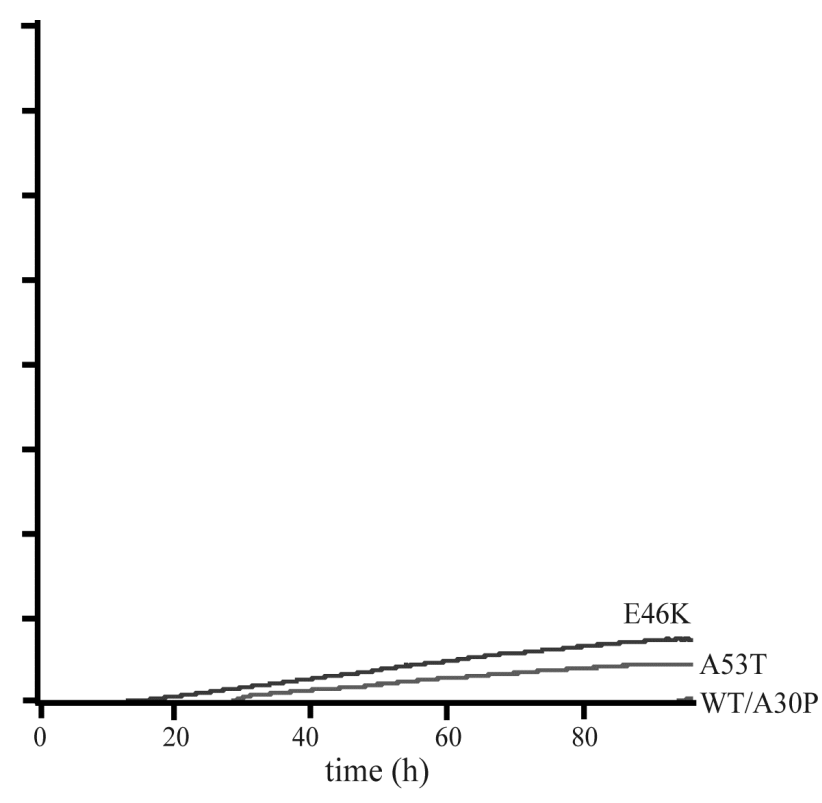

(b)

Figure 1. Amyloid fibril formation of the wild-type, A30P, E46K and A53T mutant $\alpha$-synucleins in the (a) absence; and (b) presence of $1 \mathrm{mM}$ DA as monitored by ThT fluorescence at $482 \mathrm{~nm}$.

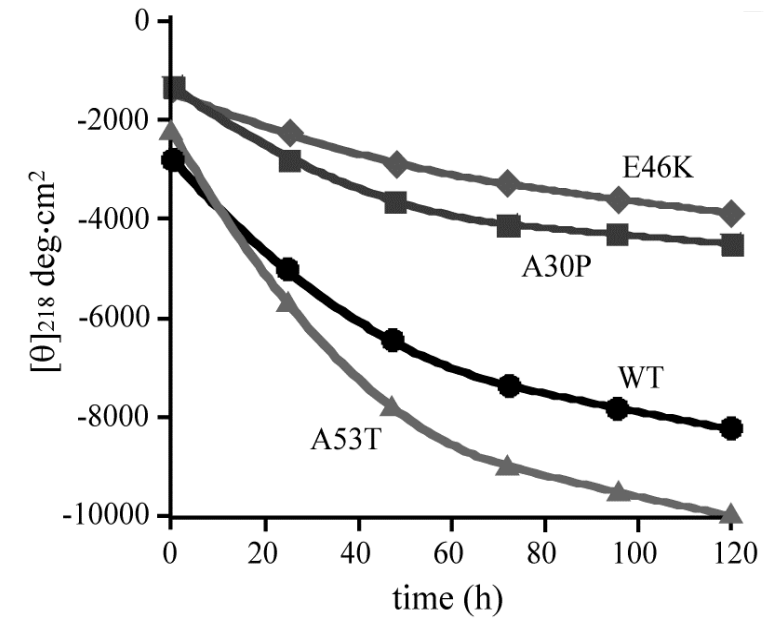

(a)

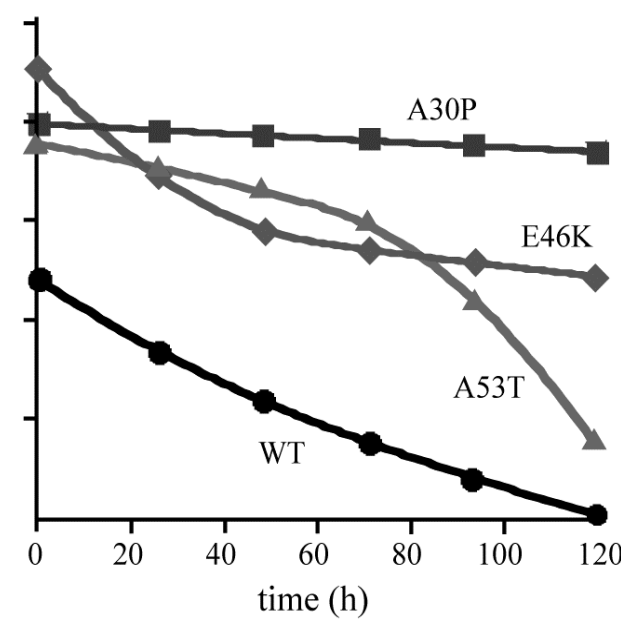

(b)

Figure 2. The plot of time courses for the molar ellipticities at wavelength $218 \mathrm{~nm}$ for the wild-type (circle), A30P (square), E46K (diamond) and A53T (triangle) mutant $\alpha$-synucleins in the (a) absence and (b) presence of 1 mM DA.

in the presence of $1 \mathrm{mM} \mathrm{DA}$ as indicated by the ThT binding assay (Figure 1(b)), it can be concluded that DA bound $\alpha$-synuclein transforms into non-amyloidogenic $\beta$-sheet-rich conformation. One of the most probable candidates for this conformation is $\alpha$-synuclein oligomers. In the previous study, our group monitored oligomerization of $\alpha$-synuclein comprising heptamer at the early stage fibrillation using the time-dependent SAXS [24]. Recently, it has been proposed that the reactive intermediates of $\alpha$-synuclein interacting with DA induce its oligomerization such as trimers $[19,20]$.

\subsection{Oligomerization of $\alpha$-Synuclein}

The effects of DA in oligomerization of $\alpha$-synuclein were monitored using size exclusion chromatography as shown in Figure 3. The monomeric wild-type and A53T $\alpha$-synucleins were eluted at 23 minutes, and the A30P and E46K were eluted at $25-26$ minutes in the absence of DA (Figures 3(a), (d), (g), (j)). Based on the calibration curve provided by Tosoh Bioscience using various standard proteins including cytochrome $\mathrm{C}(12.4 \mathrm{kDa})$, ribonuclease A (13.7 kDa) and myoglobin $(17.8 \mathrm{kDa})$, these retention times are shorter than expected. This is due 
DA: $0 \mathrm{mM}$

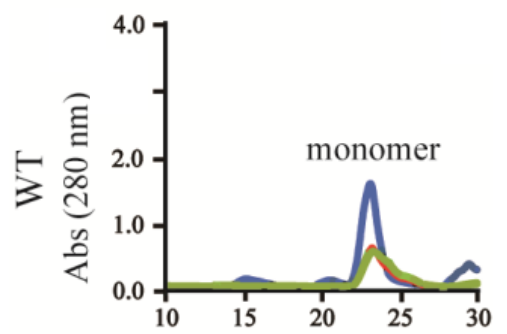

(a)

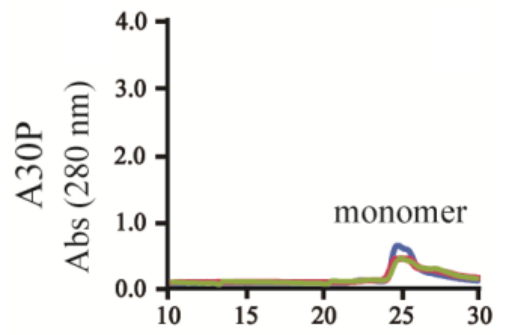

(d)

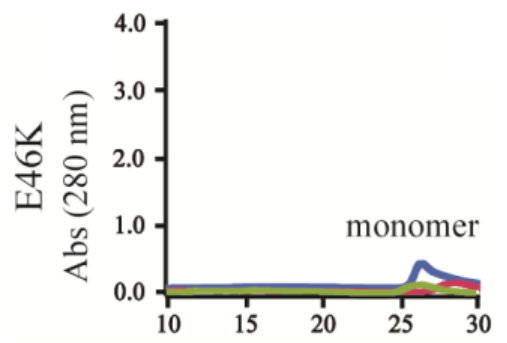

(g)

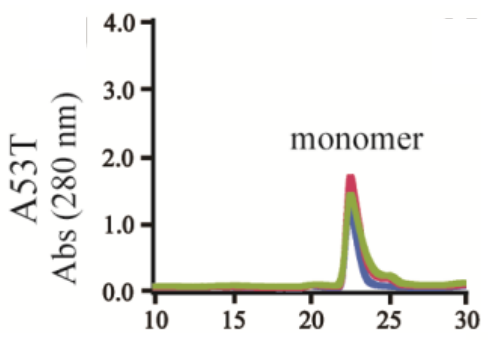

(j)
DA: $1 \mathrm{mM}$

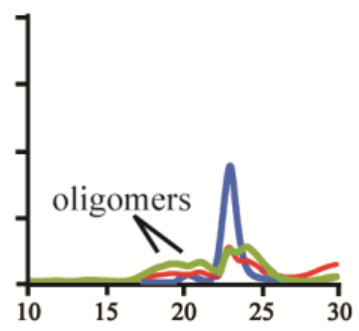

(b)

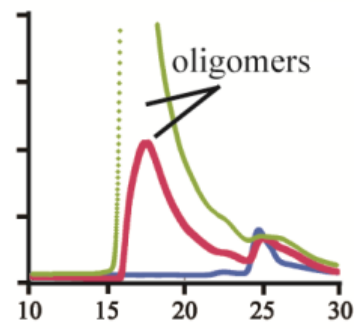

(e)

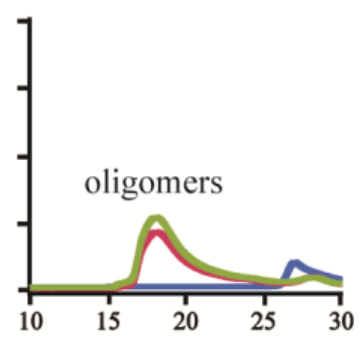

(h)

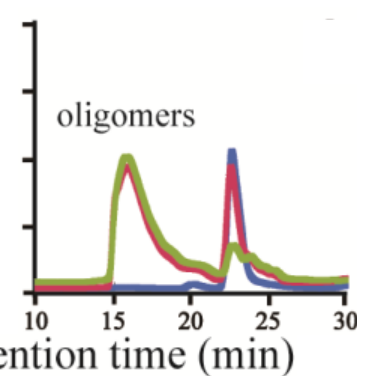

(k)
DA: $10 \mathrm{mM}$

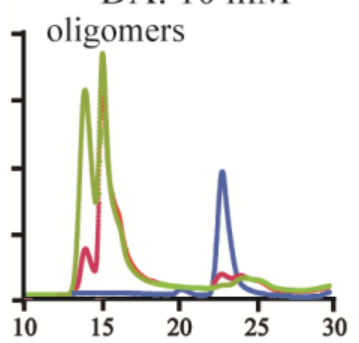

(c)

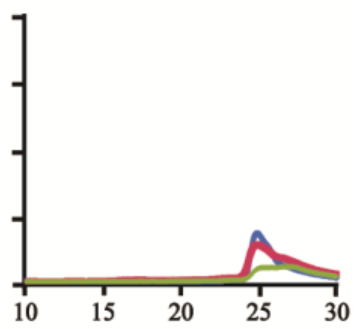

(f)

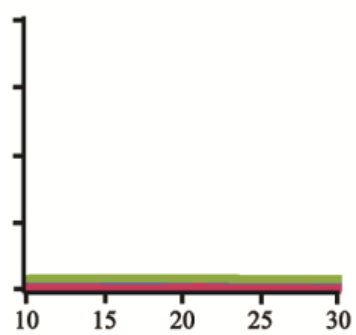

(i)

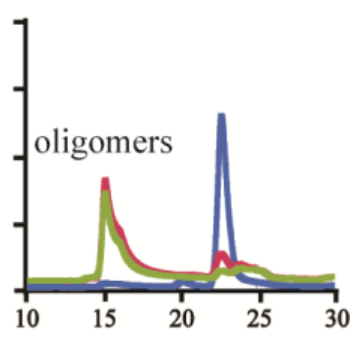

(1)

Figure 3. Size exclusion chromatography of (a)-(c) the wild-type, (d)-(f) A30P, (g)-(i) E46K and (j)-(l) A53T mutant $\alpha$-synucleins in the absence and presence of 1 and $10 \mathrm{mM} \mathrm{DA}$ at the incubation times of 0 $\mathrm{h}$ (blue), $72 \mathrm{~h}$ (red) and $120 \mathrm{~h}$ (green). The monomer peaks are labeled in each chromatogram obtained in the absence of DA, and the oligomer peaks are labeled in other chromatograms obtained in the presence of DA.

to the fact that the monomeric wild-type and mutants are more extended or unstructured than those standard proteins used for preparation of the calibration curve. The difference in retention times among the wild-type and mutants can be attributed to the deterioration of precolumn used for separation. In the chromatograms of the wild-type $\alpha$-synuclein, several high molecular weight oligomers with retention times from $18 \mathrm{~min}$ to $22 \mathrm{~min}$ were observed in the presence of $1 \mathrm{mM} \mathrm{DA}$ at 72 and 120 hour incubations (Figure 3(b)). Their molecular weights were estimated to be in the range of ca. $65-150 \mathrm{kDa}$ using the aforementioned calibration curve. The presence of these high molecular weight spieces confirms the DA induced oligomer formation for $\alpha$-synuclein. The high molecular weight oligomers were also observed in the presence of $10 \mathrm{mM}$ DA with larger peak intensities, implying more oligomer formation (Figure 3(c)). For A30P and E46K, oligomers eluting around $18 \mathrm{~min}$ were observed in the 
presence of $1 \mathrm{mM}$ DA (Figures 3(e) and (h)), while these oligomers disappeared in the presence of $10 \mathrm{mM}$ DA (Figures 3(f) and (i)). During the process of fibrillation, we have monitored protein samples using SDSPAGE and observed that the excess amount of DA promoted a fast degradation of $\alpha$-synuclein. Although a similar tendency was observed for A53T, both monomer and oligomers were still detected in the presence of 10 mM DA (Figure 3(l)). This could be attributed to the differences in the initial amount of monomer protein subjected to the fibrillation. It is as if degradation of proteins and formation of oligomers were competing events. Nonetheless, the results of size exclusion chromatography unambiguously indicate that the various molecular weight oligomers were induced for the wild-type $\alpha$ synuclein and its three mutants in the presence of $1 \mathrm{mM}$ DA. In the singular value decomposition (SVD) analysis using the time-dependent SAXS data, it was suggested that heptamers were formed in the transient intermediate state [24]. The molecular weight of heptamer is ca. 100 $\mathrm{kDa}$, which is in the range of the molecular weight of oligomers estimated by a size exclusion chromatography. In the analysis by Rekas et al., it was shown that the wild-type $\alpha$-synuclein formed various species of oligomers, such as dimmer, trimmer and high molecular weight oligomers [20]. The sedimentation velocity analysis for the eluted fraction of size exclusion chromatog-

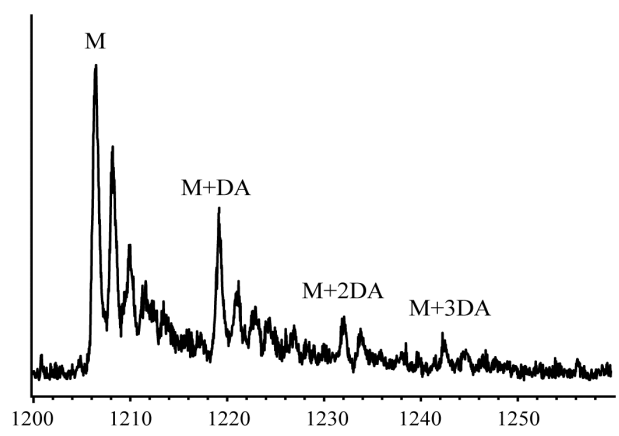

(a)

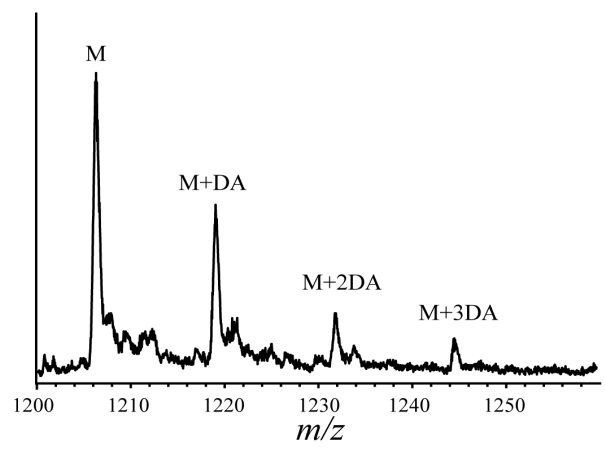

(c) raphy indicated that DA-treated wild-type $\alpha$-synuclein could have formed the maximum of 26-mers [20]. The distributions of the molecular weight of DA-induced oligomers observed in the present study are consistent with those reported by Rekas et al. [20].

\subsection{Complex Formation of Dopamine and $\alpha$-Synuclein}

In order to clarify the detailed interactions between $\alpha$-synuclein and DA at the initial stage of fibrillation, the same samples used for chromatography analysis were also subjected to MS spectrometry. Envelopes of ions for the wild-type $\alpha$-synuclein between $\mathrm{m} / \mathrm{z} 852$ and 2412, corresponding to +17 through +6 charged states monomer, were observed (data not shown). The expanded spectra between $m / z 1200$ and 1260 for all $\alpha$-synuclein species are shown in Figure 4. The envelopes of ion peaks between $\mathrm{m} / \mathrm{z} 1206$ and 1210 corresponded to the monomeric wild-type $\alpha$-synuclein ( $m w=14460)$ with +12 charged state (Figure 4(a)). In this envelope, the ion peaks consisted of the free $\alpha$-synuclein $[\mathrm{M}+12 \mathrm{H}]^{12+}$, and its sodium adducts $[\mathrm{M}+11 \mathrm{H}+\mathrm{Na}]^{12+}$ and $[\mathrm{M}+10 \mathrm{H}+2 \mathrm{Na}]^{12+}$. In the presence of DA, the ion peaks corre- sponding to the complexes of $\alpha$-synuclein and DA were observed for the wild-type $\alpha$-synuclein (Figure 4(a)) and its mutants (Figure 4(b)-(d)). The maximum of three DA molecules

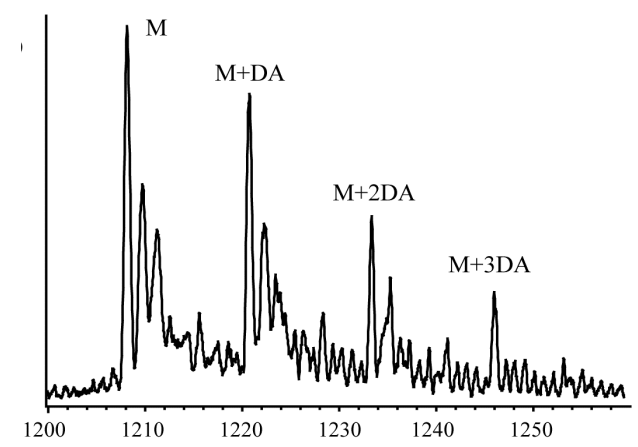

(b)

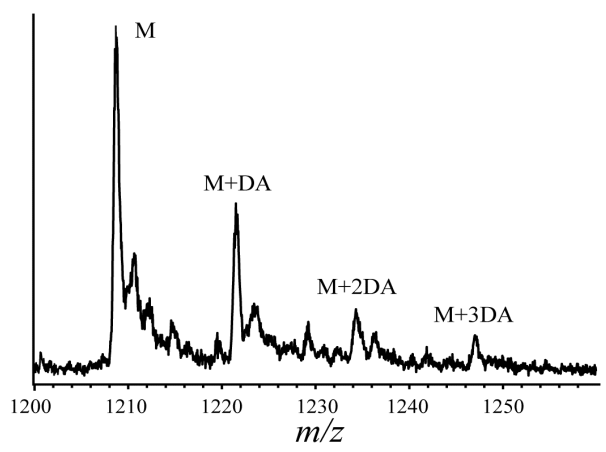

(d)

Figure 4. ESI-MS and LTQ-Orbitrap FT-mass spectra of (a) the wild-type; (b) A30P; (c) E46K; and (d) A53T mutant $\alpha$-synucleins in the presence of $1 \mathrm{mM} \mathrm{DA}$. The measurements were carried out after the addition of DA without incubation. 
were detected to bind with one molecule of $\alpha$-synuclein. The ion peaks of the complexes comprising $\alpha$-synuclein and DA (MW $=153$ ) were identified using the sample solutions without any incubation. This implies that there is a strong affinity between an intact $\alpha$-synuclein and DA. Although several groups reported observations of oligomers comprising $\alpha$-synuclein with four oxidized Met [19-21], here we have shown that non-oxidazed form of $\alpha$-synucleins had an ability to non-covalently associate with DA. These results suggest that there could be multiple pathways in oligomerization of $\alpha$-synuclein including DA. In the further study, it is necessary to clarify whether the complexes identified in our ESI-MS study is the initial compound leading to oligomers.

In the size exclusion chromatography experiments, an extinction coefficient of DA at $280 \mathrm{~nm}$ was turned out to be much larger than that of $\alpha$-synuclein. As can be seen from Figure 3, the absorbance of oligomeric species was much larger than that of monomers. This might indicate the fact that oligomers observed in these experiments were actually complexed with several DA molecules. In a working model of the fibrillization pathway of $\alpha$-synuclein, disordered monomeric $\alpha$-synuclein oligomerizes to form a heterogeneous population of $\beta$-sheet rich protofibrils, which consist of pore-like structures [26]. Since current dogma suggests that oligomeric $\alpha$-synuclein intermediates may represent the most toxic species [27], the formation of oligomeric $\alpha$-synuclein species needs to be characterized in further detail. The present results are expected to provide clues for identification of $\alpha$-synuclein oligomers.

\section{CONCLUSION}

In the present study, propensities to form oligomers at the initial stage fibrillation were studied using size exclusion chromatography and various biophysical techniques, and interactions between $\alpha$-synuclein and DA were observed using ESI-MS. In the analysis using size exclusion chromatography, it was shown that $\alpha$-synuclein oligomerizes to higher molecular weight species in the presence of DA. These oligomers are structurally different from amyloid fibrils, as confirmed by thioflavin $\mathrm{T}$ and $\mathrm{CD}$ analysis. The results of ESI-MS indicate that an intact $\alpha$-synuclein had an ability to bind with three molecules of DA at the initial stage. Based on these observations, it can be considered that formation of complexes comprising an $\alpha$-synuclein and three molecules of DA could be a critical for triggering oligomerization.

\section{ACKNOWLEDGEMENTS}

This study was supported by a Grant-in-Aid for Scientific Research (No. 17550085 for S.S and 21550092 for M.T.) from the Ministry of
Education, Culture, Sports, Science, and Technology.

\section{REFERENCES}

[1] Uéda, K., Fukushima, H., Masliah, E., Xia, Y., Iwai, A., Yoshimoto, M., Otero, D.A., Kondo, J., Ihara, Y. and Saitoh, T. (1993) Molecular cloning of cDNA encoding an unrecognized component of amyloid in Alzheimer disease. Proceedings of the National Academy of Sciences, 90, 1128211286. doi: $10.1073 /$ pnas.90.23.11282

[2] Weinreb, P.H., Zhen, W., Poon, A.W., Conway, K.A. and Lansbury, P.T. (1996) NACP, a protein implicated in Alzheimer's disease and learning, is natively unfolded. Biochemistry, 35, 13709-13715. doi:10.1021/bi961799n

[3] Davidson, W.S., Jonas, A., Clayton, D.F. and George, J.M. (1998) Stabilization of $\alpha$-synuclein secondary structure upon binding to synthetic membranes. Journal of Biological Chemistry, 273, 9443-9449. doi:10.1074/jbc.273.16.9443

[4] George, J.M., Jin, H., Woods, W.S. and Clayton, D.F. (1995) Characterization of a novel protein regulated during the critical period for song learning in the zebra finch. Neuron, 15, 361-372. doi:10.1016/0896-6273(95)90040-3

[5] Iwai, A., Masliah, E., Yoshimoto, M., Ge, N., Flanagan, L., De Silva, H.A., Kittel, A. and Saitoh, T. (1995) The precursor protein of non-A beta component of Alzheimer's disease amyloid is a presynaptic protein of the central nervous system. Neuron, 14, 467-475. doi:10.1016/0896-6273(95)90302-X

[6] Ma, Q.L., Chan, P., Yoshii, M. and Uéda, K. (2003) $\alpha$-synuclein aggregation and neurodegenerative diseases. Journal of Alzheimer's Disease, 5, 139-148.

[7] Yu, S., Li, X., Liu, G., Han, J., Zhang, C., Li, Y., Xu, S., Liu, C., Gao, Y., Yang, H., Uéda, K. and Chan, P. (2007) Extensive nuclear localization of $\alpha$-synuclein in normal rat brain neurons revealed by a novel monoclonal antibody. Neuroscience, 145, 539-555. doi:10.1016/j.neuroscience.2006.12.028

[8] Polymeropoulos, M.H., Lavedan, C., Leroy, E., Ide, S.E., Dehejia, A., Dutra, A., Pike, B., Root, H., Rubenstein, J., Boyer, R., Stenroos, E.S., Chandrasekharappa, S., Athanassiadou, A., Papapetropoulos, T., Johnson, W.G., Lazzarini, A.M., Duvoisin, R.C., Di Iorio, G., Golbe, L.I. and Nussbaum, R.L. (1997) Mutation in the $\alpha$-synuclein gene identified in families with Parkinson's disease. Science, 276, 2045-2047. doi:10.1126/science.276.5321.2045

[9] Kruger, R., Kuhn, W., Muller, T., Woitalla, D., Graeber, M., Kosel, S., Przuntek, H., Epplen, J.T., Schols, L. and Riess, O. (1998) Ala30Pro mutation in the gene encoding alpha-synuclein in Parkinson's disease. Nature Genetics, 18, 106-108.

[10] Zarranz, J.J., Alegre, J., Gomez-Esteban, J.C., Lezcano, E., Ros, R., Ampuero, I., Vidal, L., Hoenicka, J., Rodriguez, O., Atares, B., Llorens, V., Gomez Tortosa, E., del Ser, T., Munoz, D.G. and De Yebenes, J.G. (2004) The new mutation, E46K, of $\alpha$-synuclein causes Parkinson and Lewy body dementia. Annals of Neurology, 55, 164-173. 
doi:10.1002/ana.10795

[11] Arima, K., Uéda, K., Sunohara, N., Hirai, S., Izumiyama, Y., Tonozuka-Uehara, H. and Kawai, M. (1998) Immunoelectron-microscopic demonstration of NACP $/ \alpha$-synuclein-epitopes on the filamentous component of Lewy bodies in Parkinson's disease and in dementia with Lewy bodies. Brain Research, 808, 93-100. doi:10.1016/S0006-8993(98)00734-3

[12] Uversky, V.N., Li, J. and Fink, A.L. (2001) Evidence for a partially folded intermediate in $\alpha$-synuclein fibril formation. Journal of Biological Chemistry, 276, 10737-10744. doi:10.1074/jbc.M010907200

[13] Uversky, V.N. and Fink, A.L. (2004) Conformational constraints for amyloid fibrillation: The importance of being unfolded. Biochimica et Biophysica Acta, 1698, 131-153.

[14] Uversky, V.N. (2003) A protein-chameleon: Conformational plasticity of $\alpha$-synuclein, a disordered protein involved in neurodegenerative disorders. Journal of Biomolecular Structure \& Dynamics, 21, 211-234.

[15] Giasson, B.I., Murray, I.V., Trojanowski, J.Q. and Lee, V.M. (2001) A hydrophobic stretch of 12 amino acid residues in the middle of $\alpha$-synuclein is essential for filament assembly. Journal of Biological Chemistry, 276, 2380-2386. doi:10.1074/jbc.M008919200

[16] Miake, H., Mizusawa, H., Iwatsubo, T. and Hasegawa, M. (2002) Biochemical characterization of the core structure of $\alpha$-synuclein filaments. Journal of Biological Chemistry, 277, 19213-19219. doi:10.1074/jbc.M110551200

[17] Hoyer, W., Cherny, D., Subramaniam, V. and Jovin, T.M. (2004) Impact of the acidic C-terminal region comprising amino acids $109-140$ on $\alpha$-synuclein aggregation in vitro. Biochemistry, 43, 16233-16242. doi:10.1021/bi048453u

[18] Conway, K.A., Rochet, J.C., Bieganski, R.M. and Lansbury, P.T. Jr. (2001) Kinetic stabilization of the $\alpha$-synuclein protofibril by a dopamine- $\alpha$-synuclein adduct. Science, 294, 1346-1349. doi:10.1126/science. 1063522

[19] Pham, C.L.L., Leong, S.L., Ali, F.E., Kenche, V.B., Hill, A.F., Gras, S.L., Barnham, K.J. and Cappai, R. (2009) Dopamine and the dopamine oxidation product 5,6-dihydroxylindole promote distinct on-pathway and off-pathway aggregation of $\alpha$-synuclein in a $\mathrm{pH}$-dependent manner. Journal of Molecular Biology, 387, 771-785. doi:10.1016/j.jmb.2009.02.007

[20] Rekas, A., Knott, R.B., Sokolova, A., Barnham, K.J., Perez, K.A., Masters, C.L., Drew, S.C., Cappai, R., Curtain, C.C. and Pham, C.L.L. (2010) The structure of dopamine induced $\alpha$-synuclein oligomers. European Biophysics Journal, 39, 1407-1419. doi:10.1007/s00249-010-0595-x

[21] Bisaglia, M., Tosatto, L., Munari, F., Tessari, I., de Laureto, P.P., Mammi, S. and Bubacco, L. (2010) Dopamine quinones interact with $\alpha$-synuclein to form unstructured adducts. Biochemical and Biophysical Research Communications, 394, 424-428. doi:10.1016/j.bbrc.2010.03.044

[22] Lee, H.J., Baek, S.M., Ho, D.H., Suk, J.E., Cho, E.D. and Lee, S.J. (2011) Dopamine promotes formation and secretion of non-fibrillar $\alpha$-synuclein oligomers. Experimental and Molecular Medicine, 43, 216-222. doi:10.3858/emm.2011.43.4.026

[23] Kamiyoshihara, T., Kojima, M., Uéda, K., Tashiro, M. and Shimotakahara, S. (2007) Observation of multiple intermediates in $\alpha$-synuclein fibril formation by singular value decomposition analysis. Biochemical and Biophysical Research Communications, 355, 398-403. doi:10.1016/j.bbrc.2007.01.162

[24] Tashiro, M., Kojima, M., Kihara, H., Kasai, K., Kamiyoshihara, T., Uéda, K. and Shimotakahara, S. (2008) Characterization of fibrillation process of $\alpha$-synuclein at the initial stage. Biochemical and Biophysical Research Communications, 369, 910-914. doi:10.1016/i.bbrc.2008.02.127

[25] Choi, W., Zibaee, S., Jakes, R., Serpell, L.C., Davletov, B., Crowther, R.A. and Goedert, M. (2004) Mutation E46K increases phospholipid binding and assembly into filaments of human $\alpha$-synuclein. FEBS Letters, 576, 363-368. doi:10.1016/i.febslet.2004.09.038

[26] Volles, M.J. and Lansbury, P.T. Jr. (2003) Zeroing in on the pathogenic form of $\alpha$-synuclein and its mechanism of neurotoxicity in Parkinson's disease. Biochemistry, 42, 7871-7878. doi:10.1021/bi030086j

[27] Tetzlaff, J.E., Putcha, P., Outeiro, T.F., Ivanov, A., Berezovska, O., Hyman, B.T. and McLean, P.J. (2008) CHIP targets toxic $\alpha$-synuclein oligomers for degradation. Journal of Biological Chemistry, 283, 17962-17968. doi:10.1074/jbc.M802283200 